\title{
Ambiances
}

anbiances Environnement sensible, architecture et espace urbain Varia | 2017

\section{Rénovation urbaine post-industrielle et mise en ambiance méditerranéenne à Kawasaki : une rédemption par le pastiche?}

Urban Renovation and Mediterranean Atmosphere in Kawasaki: Pastiche as a Redemption?

Denis Bocquet

\section{OpenEdition}

Journals

Édition électronique

URL : http://journals.openedition.org/ambiances/858

DOI : $10.4000 /$ ambiances. 858

ISSN : 2266-839X

Éditeur :

Direction Générale des Patrimoines - DAPA - MCC, UMR 1563 - Ambiances Architectures Urbanités (AAU)

Référence électronique

Denis Bocquet, «Rénovation urbaine post-industrielle et mise en ambiance méditerranéenne à Kawasaki : une rédemption par le pastiche? ", Ambiances [En ligne], Varia, mis en ligne le 20 juillet 2017, consulté le 21 avril 2019. URL : http://journals.openedition.org/ambiances/858 ; DOI : 10.4000/ ambiances.858

Ce document a été généré automatiquement le 21 avril 2019.

\section{c) (i) $९$}

Ambiances is licensed under a Creative Commons Attribution-NonCommercial-NoDerivatives 4.0 International License. 


\title{
Rénovation urbaine post- industrielle et mise en ambiance méditerranéenne à Kawasaki : une rédemption par le pastiche?
}

\author{
Urban Renovation and Mediterranean Atmosphere in Kawasaki: Pastiche as a \\ Redemption?
}

Denis Bocquet

Kawasaki, une ville d'environ 1,5 millions d'habitants, dans la préfecture de Kanagawa au Japon, est située entre Tokyo et Yokohama, les deux principales villes du pays, avec lesquelles elle forme l'armature de l'agglomération du Grand Tokyo (Kanto et Yamanashi), généralement considérée comme la plus importante du monde, avec plus de 35 millions d'habitants (Florida, Gulden \& Mellander, 2008). Neuvième ville du pays par sa population, Kawasaki est, depuis une trentaine d'années, confrontée à divers défis relevant autant de l'identité urbaine que de la relance d'une économie stagnante (Edington, 1991). La ville s'est ainsi engagée, depuis la fin des années 1990, dans une politique ambitieuse de repositionnement à la fois urbain et économique. Celle-ci a eu pour principaux vecteurs, d'une part, l'écologie urbaine, dont Kawasaki est devenue un exemple internationalement reconnu, à la pointe de la réflexion sur la durabilité, avec une posture originale et novatrice d'intégration de l'élément urbain dans les cycles de l'écologie industrielle (Van Berkel, 2009), et, d'autre part, l'innovation technologique, avec l'installation dans les friches industrielles et ferroviaires situées entre le centre-ville et la mer, de divers centres et techno-parcs dédiés à la recherche de pointe, en liaison avec les grands acteurs économiques du pays (Bass, 1998; Miyamoto, 2012). La ville est ainsi largement parvenue à conjurer le risque du déclin économique. Mais les solutions choisies n'avaient que peu de prise sur une possible renaissance d'un centre-ville toujours marqué par les friches industrielles et la césure morphologique des voies de chemin de fer (Eto, 2005). Le vieillissement de la population, et ses effets d'isolement, rendait encore 
plus prégnant le besoin d'une revitalisation du centre-ville, tant pour l'économie directement que pour les caractères relevant de la vie quotidienne.

Les études urbaines au Japon ont montré combien cet enjeu était prégnant, en général, dans les villes de l'archipel (Takano, 2002; Roberts, 2002; Sorensen, 2006). L'effort de relance de la ville, donc, s'il était resté confiné aux espaces marginaux des anciennes zones industrielles entre les voies de chemin de fer et l'océan, ou à la recherche de davantage de vertu environnementale dans le traitement des déchets, n'aurait guère eu d'effet sur les caractères d'urbanité de Kawasaki sans la mise en place d'un vaste programme de revitalisation du centre. Dans le contexte d'une ville longtemps perçue comme le simple dortoir anonyme et sans âme d'employés du secteur des services à Tokyo ou Yokohama, ou d'ouvriers des immenses zones industrielles s'étendant le long du Pacifique, de l'aéroport d'Haneda au port de Yokohama en passant par les grands polders de Kawasaki dans la baie de Tokyo au débouché du fleuve Tama, sur lesquels s'était appuyée la reprise industrielle japonaise de l'après Seconde Guerre mondiale (Flüchter, 1975), il a toujours été délicat pour la ville de s'affirmer en tant que telle. Excentrés par rapport au noyau urbain matérialisé par la gare centrale, les quartiers résidentiels ouvriers, comme ceux plus aisés, ne créaient qu'une fréquentation de passage, entre la descente des trains de banlieue ou des métros et les diverses gares routières organisant la desserte locale. De la sorte, toute véritable identité urbaine a toujours été difficile à établir et, entre banlieue et ville industrielle, Kawasaki a longtemps souffert d'un profond déficit de lisibilité urbaine. La documentation urbanistique relative aux années 1990, qu'elle émane de la mairie, des promoteurs ou de la presse locale, se fait souvent l'écho de ce déficit de caractère urbain pour la ville.

3 Cet état de fait a des racines historiques. Si l'urbanisation, en effet, avait commencé dès la fin $\mathrm{du} 19^{\mathrm{e}}$ siècle le long de la ligne de chemin de fer du Tokaido, le phénomène était d'abord resté limité. Le reste de ce qui constitue la ville aujourd'hui n'était encore que villages épars. Dévastée comme la presque totalité de la région aux lendemains du grand tremblement de terre du Kanto, qui avait fait le $1^{\text {er }}$ septembre 1923 plus de 130000 victimes dans la région métropolitaine de Tokyo, la ville a été refondée en 1924. Mais là encore, elle est restée marginale par rapport au grand programme de reconstruction appliqué à Tokyo dans le cadre d'une exaltation nationaliste intégrant rhétorique de la catastrophe et idéologie d'un urbanisme servant de miroir au culte de la puissance, en cours dans le pays dans les années 1930 (Schencking, 2002 ; Sorensen, 2002). A Kawasaki, la reconstruction a été plus pragmatique, prosaïque, fonctionnelle. Il en a été de même à la suite des bombardements américains de 1945, dans lesquels sa position de nœud ferroviaire et de zone industrielle portuaire avait fait de la ville une cible de choix. Cette situation, conjuguée à l'attentisme institutionnel d'un gouvernement japonais qui ne confère le titre officiel de ville à Kawasaki qu'en 1972, a contribué à retarder l'émergence d'une forte identité locale pouvant se fonder sur les caractères d'une urbanité partagée (Hein \& Pelletier, 2006). Bien sûr l'urbanité, en tant que valeur au croisement des qualités de l'espace bâti et de la capacité des habitants à faire vivre cet espace et à se l'approprier (Beuscart \& Peerbaye, 2003) ne dépend pas uniquement de la reconnaissance institutionnelle de la qualité de ville. Mais ce retard en dit long cependant sur le délaissement par les divers échelons des autorités de planification de l'espace central d'une ville de cette taille, ce qui n'avait pas empêché les habitants de développer un fort sentiment civique d'appartenance, manifesté, par exemple, lors des nombreuses fêtes locales prenant la rue comme cadre d'expression. Pleinement intégrée au réseau de 
transport urbain et suburbain de la région de Tokyo, la ville, en somme, peinait à trouver sa place (Hirooka, 2000). L'étalement urbain considérable qui a marqué la fin du $20^{\mathrm{e}}$ siècle (Sorensen, 1999) n'a pas contribué, naturellement, à corriger ce déficit d'image et de substance, avec le développement en direction des collines de quartiers entiers de petits pavillons. Kawasaki a de plus encore été à la marge lorsqu'il s'est agi de faire de Tokyo une ville mondiale (Fujita, 1991 ; Machimura, 1992), se reflétant dans les nouvelles valeurs d'esthétique urbaine et de consumérisme de la globalisation : loin de participer à cet élan, elle a au contraire été reléguée dans l'imaginaire métropolitain au statut de ville sans âme et sans véritable centre. L'insistance marquée sur la nécessité d'intensifier la vie urbaine dans quelques quartiers emblématiques de la mégapole, autour du secteur de la finance, du commerce et de l'économie du divertissement (Sorensen, 2000), a paradoxalement contribué à rendre les espaces périphériques, et une ville comme Kawasaki, plus marginaux du point de vue de l'animation urbaine, mais aussi de la dynamique créative en général. Kawasaki, dans l'esprit de ses édiles, se devait donc non seulement de corriger cette tendance qui faisait d'elle chaque jour un peu plus une banale périphérie, tout en s'appuyant sur une substance urbaine déjà historiquement déficitaire.

4 C'est dans ce contexte que l'invention que l'on pourrait qualifier de post-moderne d'une identité et d'une image de centralité presque de toutes pièces a pris pour la ville une pertinence certaine aux yeux tant de la municipalité que des investisseurs, notamment les sociétés immobilières liées au groupe Toshiba, qui possédait encore de nombreux terrains dans les friches urbaines. Comme l'a montré Edward Soja pour le cas de Los Angeles (Soja, 1995 ; Ellin, 1999), urbanisme dit post-moderne et recomposition négociée entre décideurs et investisseurs des imaginaires urbains sont souvent liés. Les études urbaines ont aussi montré combien la dimension de thématisation et de pastiche (Voyé, 2003) étaient des instruments de revitalisation urbaine et commerciale promus de manière internationalisée par d'efficaces firmes et agences (Gottdiener, 1997 ; Lukas, 2007). Même si à Kawasaki, résidents des quartiers aisés et ouvriers avaient depuis plusieurs décennies un fort sentiment d'appartenance locale, et si l'identité de chaque îlot résidentiel était puissamment ancrée dans la vie quotidienne des habitants (Besto, 1989; Berque, 1993 ; Hein, 2008), manquait un caractère de centralité qu'une telle combinaison allait tenter de susciter. Cela, naturellement, pose de nombreuses questions, à la fois autour de ce cas, mais aussi quant à la réflexion sur les notions mêmes d'urbanité et de revitalisation urbaine.

5 La présente étude est fondée sur une recherche de terrain ayant compris des entretiens avec les responsables municipaux de l'urbanisme, l'étude des documents d'urbanisme publics disponibles au sujet des plans des années 1980 à 2010 et les projets soumis par les équipes d'architectes et d'urbanistes lors de diverses phases de concours et consultations publiques ainsi que des phases d'observation in situ, avec des entretiens avec les usagers. Elle tend à poser la question, en termes d'urbanité, de la rédemption par le pastiche, et par le choix de la consommation de masse aussi bien que de formes urbaines pastiches, des limites des transformations de la ville mises en œuvre dans les années 1980 et 1990. La notion d'ambiance (Augoyard, 1995) est ici convoquée afin de tester une autre manière de lire les processus de transition urbaine. Celles-ci, en effet, ont été davantage lues en termes de géographie économique, d'urbanisme ou de politique de la ville. Explorer le lien entre imaginaires transplantés et toutes ces dimensions, qui demeurent fondamentales, permet de mettre en discussion les mécanismes mêmes de la transition. 
6 Kawasaki avait constitué, depuis 1981, un véritable laboratoire au Japon pour la transition de la ville industrielle vers la ville des services et des technologies de l'information (Newstead, 1989; Graham, 1992). Alors qu'encore en 1960 un plan métropolitain pour l'industrie lourde affectait considérablement son espace, dès 1969 il avait été décidé de rompre avec ce choix et de privilégier les industries légères. En 1981, le plan Kawasaki 2001 mettait l'accent sur les technologies de l'information et les services. Mais ce plan d'urbanisme ne pensait pas l'urbanité : on s'en remettait à l'équipement technique pour penser la transition, ce que confirmait un nouveau plan, en 1986, organisant le remplissage des espaces délaissés de l'industrie lourde par ce qu'on appelait déjà les knowledge-based industries. La information city qui était censée en naître n'était pas pensée en termes urbains, mais uniquement selon une logique technicienne et économique. L'année suivante, les deux plans fusionnèrent (Newstead, 1989), laissant place au concept de Campus-City (Kumata, 1990). C'est le début d'une prise de conscience des lacunes des efforts précédents : le centre-ville doit devenir le modèle d'une smart-city du futur et les hautes technologies sont explicitement désignées et théorisées comme instruments de revitalisation urbaine. Mais c'est davantage le câblage de la ville par la fibre optique qui est mis en avant, que d'hypothétiques efforts d'urbanité, et même d'urbanisme (Kawakami, 1988). Un début de changement, déterminant pour la compréhension de la phase analysée ici, est à observer cependant dès cette année 1987. La municipalité lance en effet un concours international d'urbanisme, auquel participent près de 200 équipes, issues aussi bien du Japon que d'Europe et des Etats-Unis ${ }^{1}$. Le premier prix est attribué à Peter Droege, enseignant au MIT, qui théorisera dans les années suivantes le concept d' intelligent environments (Droege, 1997). C'est dans ce concours qu'apparaît pour la première fois dans l'histoire urbaine de Kawasaki le concept de Plaza. C'est de ce moment qu'on peut dater le début du recours à la mise en ambiance comme stratégie de rénovation urbaine à Kawasaki. L'ambiance est pour la première fois conçue comme support et secours d'urbanité destiné à corriger l'aridité de la transition pensée auparavant autour de la technologie. D'emblée, cette ambiance est associée aux fonctions commerciales et récréatives, vues aussi comme source potentielle de rédemption. Pour Droege, dont le discours demeure cependant ancré dans la dimension technologique, créer des places urbaines, entourées de neighbourhood offices reliés par fibre optique au cœur de Tokyo, pourra conférer à la ville le caractère urbain qui lui manque (Droege, 1989). D'autres équipes utilisent le concept d'intelligent plaza (Reinermann, 1986). On voit là clairement l'intersection précoce entre genèse de la smart city japonaise et tournant de la mise en ambiance à consonance méditerranéenne. C'est sur ces bases que le réenchantement des urbanités nippones va se situer, au confluent du câblage et de la création d'ambiances. Un véritable cahier des charges dérive pour les autorités locales de cette étape, qui sera crucial dans la maturation des décisions ultérieures. En luttant contre les déplacements pendulaires et en sédentarisant les travailleurs du tertiaire, les urbanistes pensent aussi aider à renforcer l'urbanité et le sens local de la community : ces mots reviennent souvent dans les conversations avec les acteurs. Suivant les préceptes formulés par Droege (mâtinés d'emprunts à diverses autres équipes ayant participé au concours), la municipalité pense que le fait que les «plazas soient connectées en ligne pour pouvoir interagir avec les autres plazas par la transmission multimédia de l'information » saura créer de la ville (Kumata, 1990). Mais les années 1990 ont montré que la rédemption post-industrielle, du moins pour ce qui concerne les caractères de l'urbanité, ne saurait émerger uniquement de cette piste-là et de la croyance en la vertu innée de la connexion pour la création d'urbanité. La plaza, en effet, au cours de cette 
phase n'est pensée que dans ses lignes générales, vaste vide sur les documents d'urbanisme, et l'ambiance n'est pas vraiment travaillée en tant que telle dans son rapport à la forme construite. Au cours des années suivantes d'ailleurs, les déplacements pendulaires se sont encore renforcés, et le plan Campus-City n'a été efficace, d'un point de vue économique, que dans les anciennes zones industrielles situées entre la ville et la mer. Le centre-ville est demeuré tout à fait défaillant quant à la vie urbaine. C'est de ce constat qu'est né dans les années 1990 un nouveau plan municipal, laissant place à une nouvelle stratégie, celle d'une tentative de rédemption urbaine par la consommation de masse et la construction de grands centres commerciaux. Le choix délibéré d'une forme urbaine pastiche s'inscrit dans ce contexte, avec le recours à des professionnels de la création d'ambiances. La plaza n'est plus seulement l'articulation rhétorique de deux impératifs, mais bien le cœur d'une nouvelle stratégie.

Deux initiatives de la fin des années 1990, prises dans le cadre d'un plan municipal de revitalisation urbaine négocié avec les investisseurs, ont donc tenté de repositionner l'image de la ville et de donner un nouveau sens du lieu à ses habitants, jusque-là trop pressés au sortir de la gare de regagner leurs logements, serrés entre le chemin de fer et les collines ou en direction du littoral. Dans cette nouvelle phase d'urbanisme, s'articulent vision institutionnelle publique, par le travail d'encadrement réglementaire et d'accompagnement planificateur de la mairie, et dévolution du travail non seulement de transformation urbaine, mais aussi d'urbanité, à des opérateurs privés et à des agences spécialisées. Il illustre aussi, pour le renouveau de la ville, la foi en la puissance économique et de création d'espace public du secteur de la consommation et des loisirs. Avec ces deux opérations, enclaves ouvertes destinées par leur caractère d'îlots d'animation à avoir un effet d'entrainement, en somme, Kawasaki entre dans une ère nouvelle de son histoire urbaine, dans une relation plus complexe qu'il n'y pourrait paraitre cependant avec la simple translation spatiale à l'échelle locale de tendances issues de la sphère de la globalisation. Ce que le recours à la notion d'ambiance permet d'éclairer, c'est l'existence d'une véritable politique de théorisation du lien entre forme construite, structure foncière, consommation et sens du lieu. Si l'éventail des solutions d'urbanisme, l'identité de certains des personnages impliqués et l'imaginaire convoqué relèvent bien d'une dimension de globalité combinée à ce qu'on pourrait définir comme la sphère architecturale, esthétique, narrative et consumériste du post-modernisme, cet exemple illustre aussi combien à chaque articulation de ces ensembles plus ou moins clairs avec le local s'opèrent des interactions originales et uniques, qui contribuent à donner en retour un caractère moins caricatural qu'on pourrait le penser à ces nouveaux lieux. Cette conjonction fait aussi de ces lieux de véritables laboratoires de l'analyse des politiques urbaines, pour lesquelles la notion d'ambiance constitue une entrée permettant la mise à plat des mécanismes et l'explicitation de leurs limites et ambiguïtés.

8 Le premier de ces projets est l'œuvre d'un architecte américain, Jon Jerde (1940-2015), à la fois marginal et insolemment productif dans la profession, en raison de sa spécialisation assumée, et même théorisée au regard de l'histoire de l'architecture et de son tournant dit post-moderne, dans le domaine de la mise en ambiance des centres commerciaux et des parcs à thèmes (Begren, 1998 ; Koolhaas, 1995). Le concepteur du Mall of America dans le Minnesota, ou de l'hôtel Bellagio à Las Vegas (Ritzer, 2001 ; Nédélec, 2012), face à tous les théoriciens de l'architecture qui fustigeaient le genre auquel il se dédiait et sa prétention à en faire une expression authentique de vie sociale et d'urbanité, a ainsi développé une approche qui a fait de lui un des représentants les plus en vue, et 
controversés, d'un urbanisme non seulement post-moderne par son positionnement esthétique, pastiche par sa forme et son ornement, et fonctionnel en rupture avec les principes du modernisme, mais encore, et paradoxalement, carrément anticonformiste par rapport aux canons partagés dans la profession (Jerde, 1988). L'architecte s'est mis au service du marketing de la production d'expériences et d'ambiances, et a décliné le concept selon toute une gamme de thèmes se référant à des sphères culturelles diverses (Filser, 2002). Jon Jerde est célèbre également pour ses cinémas post-modernes dans lesquels, en quelque sorte, le spectacle est aussi dans la salle, avec la création d'un univers spécifique censé créer une ambiance ajoutant à l'expérience du spectateur (Friedberg, 1991). Pour ses interventions urbaines, c'est la recréation d'une authenticité factice qui caractérise son travail, avec à chaque fois cependant la volonté de créer des espaces qui prennent vie (Benedikt, 2001). En 2008, dans une sorte de métaphore de l'histoire de l'urbanisme depuis 1945, Jon Jerde a été chargé de la régénération urbaine du centre de Coventry (Joseph-Lester, 2010), une ville bombardée pendant la Seconde Guerre mondiale dont le rapport au paradigme de la reconstruction a traversé depuis toutes les étapes de l'évolution de la théorie urbaine et du rapport entre architecture, idéologie et idée de ville (Tiratsoo, 1990). Au cours des années 1990 et 2000, l'Asie a aussi constitué pour lui le terrain d'une expression privilégiée, à l'abri peut-être de la critique d'ordre presque civilisationnel qui l'accablait en Europe et parmi l'élite architecturale américaine (Leibowitz, 2004). Il a ainsi construit de nombreux centres commerciaux en Chine. Jon Jerde, en somme, a été dans le monde un des grands tenants de l'intervention urbaine d'enclave par la dévolution au commerce d'un espace thématisé (Kärrholm, 2012; De Châtel \& Hunt, 2003). Sa touche personnelle était la création d'un imaginaire au service de la consommation et de l'expérience des loisirs, avec une incidence espérée sur la vie donnée aux lieux ainsi exaltés, mais aussi sur l'intensité de la consommation (Jascke, 2001). C'est précisément ce que recherchait l'investisseur Kawasaki Misu Co. Ltd., déjà opérateur de multiplex cinématographiques et du complexe à remplacer, le Cinecittà de Kawasaki, construit dans les années 1980 à la place d'un parc aquatique, lui-même ayant remplacé en 1967 une ancienne usine. Créer une atmosphère thématisée en fonction d'un univers de référence totalement hors de propos a priori, mais devenant pertinent en termes d'expérience vécue et de communion consumériste, telle était ainsi la gageure à Kawasaki, selon une version assez libre, mais précisément élaborée, du concept de Zeitgeist que Jerde se plaisait à cultiver (Jerde, 1998).

Dans cette ville, étape parmi d'autres dans la conquête commerciale par son agence des espaces commerciaux asiatiques, sa solution pour transformer les abords essoufflés de la gare, du côté de sa sortie Est, et redonner vie à un complexe que son opérateur, en liaison avec des autorités municipales souhaitant créer de l'espace public, jugeait démodé, a été d'y transposer une sorte de village toscan : La Cittadella. Le complexe, dont la construction a été décidée à la fin des années 1990, a été inauguré en 2003. De la toponymie à la morphologie, tout y converge pour créer l'ambiance d'un village toscan. Sauf qu'il s'agit au fond d'un grand multiplex de cinémas, avec commerces, discothèques et restaurants. Sauf aussi qu'il s'agit d'un bout de ville, le centre de la ville même. 155 logements font aussi partie du programme. Partant d'une sorte de corso piétonnier, la via Cinecittà, une petite rue pavée, monte selon un parcours hélicoïdal en direction d'une petite chapelle, la Cappella Sant'Angelo. Le long de la rue, des magasins proposent des articles d'inspiration méditerranéenne, ou du moins touristique et exotique. Dans la chapelle, sont célébrés des mariages sous l'égide d'une entreprise proposant des solutions globales. L'assistance peut jeter des grains de riz sur la mariée sur la petite place pavée qui s'étend devant la 
chapelle, avant d'aller célébrer la nouvelle union (matrimoniale et culturelle) dans le restaurant italien qui fait face à la chapelle sur la place. Dans la citadelle, les évocations symboliques de l'Italie sont omniprésentes: des oliviers plantés çà et là aux petits escaliers reliant les différents niveaux du complexe, ou cette fontaine d'inspiration antique, pastichant celles qui provoquent à Rome l'émerveillement du passant, comme sur la via Giulia. Sur la façade principale, dans la rue piétonne qui traverse les lieux, deux immenses publicités pour Alitalia et Alfa Romeo font partie de l'imagerie plus que véritablement de l'offre commerciale. Mais l'élément à la puissance évocatrice la plus frappante relève du chromatisme, avec de toute part une saturation de l'horizon visuel par la couleur ocre Terra di Siena. La sonorisation de l'espace viaire (au Japon une chose courante, ainsi que dans les parcs à thème) s'abstient cependant de références méditerranéennes, au profit d'une stimulation musicale de la culture cinématographique des visiteurs, poussés après la flânerie à utiliser les lieux pour ce qu'ils sont aussi : un espace de consommation de films américains ${ }^{2}$. Car malgré la référence à Cinecittà (pour des raisons juridiques liées à la renaissance de l'original romain, le cinéma s'appelle Città, ce qui pour une oreille japonaise demeure une évocation pertinente du mythe), ce multiplex de cœur de ville se voulant village ne projette que des blockbusters qui ne sont méditerranéens que lorsque James Bond chevauche en moto-cross les toits du Grand Bazar d'Istanbul, ou que Jason Bourne se fait oublier sur une île des Cyclades des services secrets déviants qui le pourchassent ${ }^{3}$. L'imagerie de Ladri di Biciclette, de Caro Diario ou de Respiro n'est même pas convoquée au secours d'une atmosphère "méditerranéenne " dont les ressorts sont ailleurs et les attendus relèvent d'un autre registre. Le Caffé Verona lui-même est d'ailleurs un Starbucks des plus conventionnels ${ }^{4}$. Mais là aussi, il faut croire que le pastiche de l'italianité caféinée à la mode du Nord-Ouest pacifique américain ne gâche pas celui qui se déploie si puissamment autour. D'ailleurs, comme en Italie, mais c'est peut-être bien là un trait d'urbanité japonaise qui se révèle dans ce bar si standardisé (car ces choses ne s'apprennent pas), on reconnaît le client et le salue amicalement comme un habitué dès sa deuxième visite.

Le second projet d'inspiration méditerranéenne qui a contribué à changer le paysage du centre-ville de Kawasaki, mais aussi d'une certaine manière son identité, se trouve de l'autre côté de la gare, et est relié au centre-ville par l'immense hall de celle-ci, dans lequel se déverse aux heures de pointe toute une théorie de trains de banlieue cadencés de telle sorte que le flux de voyageurs franchissant les portillons ne cesse jamais. Se trouvait depuis 1908 et jusqu'en 2000 sur ce terrain tout à fait central dans la ville, une usine du groupe Toshiba, qui, à son apogée, a employé plusieurs milliers d'ouvriers. Cette usine, progressivement fermée entre la fin des années 1970 et la fin des années 1990, a même été, pendant presque un siècle, le symbole urbain du caractère industriel de Kawasaki. Mais avec la mutation sectorielle entamée par le groupe, d'une part, et avec l'émergence au sein de la municipalité, d'une volonté de favoriser la régénération urbaine par un nouvel usage pour ces lieux, d'autre part, il est devenu évident que l'ancienne usine allait désormais devenir le symbole d'un changement d'identité pour la ville. Dès avant la fermeture, s'est de la sorte posée avec acuité la question de la revitalisation urbaine post-industrielle dans le cadre du plan municipal. Le cas de Kawasaki n'est bien sûr pas isolé, et à la même époque, de nombreuses villes industrielles entreprenaient des démarches similaires. De Pittsburgh, avec la réinvention de la ville sur la base d'une renaissance du centre et du développement d'activités de hautes technologies sur les lieux des anciennes usines sidérurgiques, à Turin, qui est parvenue à sortir par le haut du déclin du secteur automobile (Capello, Carigno \& Vignolo, 2010), en passant par Berlin où 
les anciennes usines Borsig ont été transformées en centre commercial (Kulke, 2001), et à Yokohama, devenu au Japon le modèle d'une revitalisation urbaine post-industrielle, les exemples ne manquent pas qui illustrent les stratégies mises en œuvres localement pour réinventer des villes en crises. Mais à Kawasaki, la spécificité a été de jouer, en sus de la dimension d'usage commercial des lieux d'une ancienne usine, sur la création ex-nihilo d'une urbanité d'ambiance d'inspiration méditerranéenne. Point d'exaltation, comme aux Borsigwerke, ou aux Officine Grandi Riparazioni, des vestiges de l'architecture industrielle. Ne reste que, cachée dans un angle mort, une borne à eau de l'ère industrielle. Pour le reste, ces lieux, rebaptisés LAZONA Plaza Kawasaki, ont été conçus comme l'écrin d'ambiance du consumérisme. Le site, qui appartient toujours aux fonds NREG Toshiba Building et Mitsui Fudosan, a été confié à la compagnie de promotion immobilière Lalaport, déjà opérateur, entre autres, d'un grand centre commercial dans la périphérie de Tokyo, qui y a fait construire un ensemble commercial de 150000 mètres carrés, comprenant plus de 300 magasins, dont de nombreuses grandes surfaces. La structure, inaugurée en 2006, a été conçue par l'architecte catalan Ricardo Bofill et s'articule autour d'une haute canopée métallique. L'espace central est constitué d'une place plantée, évoquant un espace public à la méditerranéenne et en stimulant l'usage. Pour le reste, le shopping mall est relativement classique. Mais l'usage des traits de caractère méditerranéens aux fins de créer une mise en ambiance est clair dès le débouché du hall de la gare, auquel la plaza est reliée : on entre dans la dimension de la détente, du ralentissement du pas, de la flânerie... de l'achat. Vu de Kawasaki, le recours à un architecte du prestige de Ricardo Bofill a servi de catalyseur au déploiement d'une rhétorique visant à la constitution d'une urbanité nouvelle, confiée dans un isolat, en termes de planification mais point de flux, au commerce privé. La portée civique locale forte en termes d'identité des lieux n'est pas absente derrière l'apparente logique purement commerciale. Le recours à Ricardo Bofill a été pensé en tant que référence culturelle autant que d'appel à une expertise technique. Le personnage n'est pourtant pas spécialement connu en Méditerranée même pour sa contribution à l'urbanité justement méditerranéenne et ses productions ont souvent déclenché d'âpres polémiques, comme à Salerne, où l'association de protection du patrimoine Italia Nostra a qualifié en 2014 un de ses projets de monstre écologique. Ricardo Bofill a aussi été vilipendé pendant de longues années pour sa vision de l'extension de Montpellier, pour ne citer qu'un exemple (Hoyaux, 1998). Mais c'est peut-être pour l'avoir déjà pastiché en Méditerranée qu'il est parvenu, dans un contexte japonais, à donner au centre commercial au moins un espace public qui fonctionne en tant que tel, sur lequel les adolescents se donnent rendez-vous, les mamans achètent des glaces à leurs enfants et quelques vieillards regardent passer la foule, assis sur une chaise à l'ombre d'un arbre. LAZONA se vante d'avoir, durant le premier mois de son ouverture, attiré plus de 10 millions de visiteurs, soit autant que Disneyland Tokyo, autre espace de la construction culturelle du factice ${ }^{5}$. Naturellement, les deux espaces, et expériences, ne sont pas comparables. Mais le succès, comme l'indiquent les statistiques d'évolution du chiffre d'affaire de la structure gestionnaire, semble durable. Pour Kawasaki, le choix d'une industrialisation de la consommation, sur le modèle singapourien, pour faciliter la sortie de la crise industrielle et dynamiser le centre-ville, semble donc avoir été couronné de résultats ${ }^{6}$. Il symbolise aussi, en évolution par rapport à la phase précédente, une très nette privatisation de l'urbanisme: c'est désormais presqu'uniquement en propriété privée qu'est repensée l'urbanité de la ville. Le commerce, plus de dix ans après l'ouverture du complexe, ne présente pas de signe de faiblesse, même si, comme à Potsdamerplatz à Berlin avec la Deutsche Bahn, la présence 
d'un immeuble de bureaux de Toshiba sur le site est une condition nécessaire à l'activité commerciale et de restauration au moment de la pause de midi. C'est l'exemple d'une régénération urbaine entraînée par les grands groupes industriels eux-mêmes et leurs branches immobilières, en conjonction avec les autorités locales autour d'un consensus créé par une fiction culturelle qui, somme toute, fonctionne sur le registre attendu: espace public piétonnier marquant l'urbanité et incitation à la consommation. En 2013, Toshiba a inauguré sur le site un nouvel immeuble de bureau de 100000 mètres carrés, renforçant encore cette logique. C'est en ce sens que le pastiche peut être vu comme une voie de rédemption urbaine, mais aussi que ses limites s'en manifestent le plus clairement, notamment autour du rapport à la sphère civique. La mise en ambiance, ainsi, apparaît dans le cas de Kawasaki, comme le pendant scénarisé d'une libéralisation de l'urbanisme.

11 La post-modernité, ou du moins une expression de ce que ce concept peut recouvrir, telle que déclinée à Kawasaki, semble cependant bien constituer davantage qu'une simple parodie, et une analyse se limitant aux ambiguïtés fondatrices de cette forme urbaine ne ferait pas justice à la complexité de la société concernée ${ }^{7}$. Apportant une réponse urbaine aux tendances lourdes de la sociologie de la consommation au Japon, et en général à la fuite du commerce dans les grandes zones commerciales adjacentes aux échangeurs autoroutiers, les deux opérations de pastiche méditerranéen, au-delà de leur aspect factice et de leur nature d'enclaves privées, véhiculent dans leur fonctionnement de réelles valeurs d'urbanité. C'est là que la lecture en termes d'ambiance peut permettre de dépasser les constations et dénonciations faites sur le seul registre du socio-économique et du morphologique. Si l'on peut discuter de la consistance des émotions apportées par les atmosphères reconstituées, ce qui semble certain est que les solutions adoptées à Kawasaki ont apporté une forme de réponse à la question de l'urbanité telle qu'elle était posée dans cette ville. Même s'il va de soi que l'espace public peine à constituer un espace civique, il demeure que, comme se plaisent à le rappeler les urbanistes municipaux lors des entretiens, le temps de présence des citadins dans l'espace public a été augmenté, de même que la consistance et l'extension de cet espace. Le décalage entre le décor d'ambiance et les pratiques des habitants est, par ailleurs, plus étroit qu'on ne pourrait le penser. Une telle démarche de création d'ambiance n'a de sens que s'il existe un substrat favorable dans la société concernée, un horizon d'attente presque, au sens défini par Hans Robert Jauss pour le champ de la critique littéraire (Jauss, 1978), dont la nature a plus à voir avec les fondamentaux de la société locale qu'on ne pourrait le penser au seul regard du décor imposé. Si le cadre, dans toute sa fausseté post-moderne, prend véritablement vie en effet, c'est grâce à l'aptitude des habitants et usagers à déployer une urbanité dont ils sont en fait porteurs eux-mêmes et à y appliquer des pratiques qui ne sont pas étrangères à leur manière de vivre. La greffe urbaine, qui ne s'est pas embarrassée de rechercher une compatibilité culturelle de premier degré, n'a pris que parce que les fondements de la vie urbaine étaient bien présents parmi le public visé, largement audelà des simples façades du consumérisme. Le référent culturel japonais n'est pas dans le décor ou la couleur, mais bien dans l'habitude des grands-parents de promener leurs petits-enfants, des groupes de jeunes gens de se retrouver, des couples de flâner, ou plus prosaïquement, des consommateurs d'être réceptifs aux injonctions consuméristes. C'est ce que montrent les entretiens in situ : l'ambiance est propice à cela au-delà du référent culturel qu'elle affiche car elle touche à des dimensions plus profondes de l'être à la ville ou à l'espace public. Les espaces commerciaux au Japon sont ainsi en général porteurs d'une dimension puissante d'urbanité, et il n'est qu'à voir comment cette âme a conquis à 
Tokyo les interstices des grandes avenues de la reconstruction d'après 1923 et 1945, en en faisant des îlots piétonniers dédiés au commerce et à la restauration, pour comprendre qu'il existait dans les pratiques locales un terrain propice. A Kawasaki, c'était d'abord, outre les couloirs souterrains du complexe de la gare, sous la forme d'une grande rue couverte et piétonne, que la vie urbaine s'était redéveloppée dans l'après-guerre. La pratique sociale liée à des espaces piétonniers ou quasi était aussi présente dans les petites rues alentour, qui, avec leur traditionnelle dévolution à la boisson et à la prostitution, constituaient, et continuent de constituer, des enclaves de lenteur entre les grandes avenues. Dans la «fausse » ville-centre près de la gare, aseptisée par rapport à cet héritage, si une « vraie » vie a pu se développer, c'est bien qu'il en existait le terreau. La dimension d'urbanité dépend de ce dont les gens sont porteurs et de la capacité d'un espace de leur permettre de l'y déployer, quel que soit le rapport de cet espace à la sémiologie prétendument intrinsèque des lieux. Les limites de cette dimension résident davantage dans l'incapacité de tels lieux calibrés et surveillés à être porteurs d'une vie civique, que dans leur rôle, ou non, de vecteurs de vie urbaine.

Quant à la dimension méditerranéenne de cette urbanité qui en est une (vie urbaine) sans en être tout à fait une (sphère civique), les débats restent ouverts, au-delà de la paradoxale et ironique sensation d'authenticité du factice conférée par l'usage que font les citoyens des lieux qui leur sont proposés et de la communion des imaginaires que les âmes bienveillantes acceptent d'y expérimenter dans une sorte d'oxymore urbain. Se sent-on en ces lieux de Kawasaki inspirés par une imagerie méditerranéenne, autant en Méditerranée qu'au Warner Village de Rome, dans une banlieue fantôme de Madrid, à Plan de Campagne près de Marseille, dans la fausse médina de Yasmine Hammamet ou au bord du faux Bosphore d'Albert Speer Jr. à Istanbul'8? Peut-être bien après tout.

\section{BIBLIOGRAPHIE}

\section{Bibliographie}

Arsel, Zeynep \& Thompson, Craig. 2004. The Starbucks Brandscape and Consumers'

(Anticorporate) Experience of Glocalization. Journal of Consumer Research. vol 31, no 3, p. 631-642.

Augoyard, Jean-François. 1995. L'environnement sensible et les ambiances architecturales. L'espace géographique. vol 24, $\mathrm{n}^{\circ}$ 4, p. 302-318.

Bass, Steven. 1998. Japanese Research Parks: National Policy and Local Development. Regional Studies. vol 32, no 5, p. 391-403.

Benedikt, Michael. 2001. Reality and Authenticity in the Experience Economy. Architectural Record . vol 189, nº 11, p. 84-87.

Berque, Augustin. 1993. Du geste à la cité : forme urbaine et lien social au Japon. Paris : Gallimard. 248 p.

Besto, Theodore. 1989. Neighborhood Tokyo. Stanford: Stanford University Press, 347 p. 
Beuscart, Jean-Samuel \& Peerbaye, Ashveen. 2003. Urbanité(s). Terrains et Travaux. vol 5, no 2, p. 3-6.

Capello, Alessandro ; Carignano, Paolo \& Vignolo, Fabio. 2010. Torino: la riqualificazione urbana dell'area Spina 4. Turin: Politecnico di Torino, $112 \mathrm{p}$.

De Châtel, Francesca \& Hunt, Robin. 2003. Retailisation: The Here, There and Everywhere of Retail. London: Routledge, 296 p.

Dodds, Klaus. 2003. Licenced to Stereotype: Geopolitics, James Bond and the Spectre of Balkanism, Geopolitics. vol 8, no 2, p. 125-156.

Droege, Peter. 1989. Technology for People. Places. vol 5, n 3, p. 50-51.

Droege, Peter. 1997. Intelligent Environments. Spatial Aspects of the Information Revolution. Amsterdam: Elsevier, 75 p.

Edington, David. 1991. Economic Restructuring in Yokohama: from Gateway Port to International Core City. Asian Geographer. vol 10, no 1, p. 62-78.

Ellin, Nan. 1999. Postmodern Urbanism, Princeton: Princeton Architectural Press, 392 p.

Eto, Hajime. 2005. Obstacles to the Emergence of High/New Technology Parks, Ventures and Clusters in Japan. Technlogical Forecasting and Social Change. vol 72, no 3, p. 359-373.

Filser, Marc. 2002. Le marketing de la production d'expérience. Décisions marketing. vol 28, p. 13-22.

Florida, Richard ; Gulden, Tim \& Mellander, Charlotta. 2008. The Rise of the Mega-Region. Cambridge Journal of Regions, Economy and Society. vol 1, nº 3, p. 459-476.

Flüchter, Winfried. 1975. Neulandgewinnung und Industrieansiedlung vor den Japanischen Küsten. Paderborn: Schönigh, 179 p.

Friedberg, Anne. 1991. Les flâneurs du mal(l) : Cinema and the Post-Modern Condition. Publications of the Modern Language Association. vol 106, no 3, p. 419-431.

Fujita, Kuniko. 1991. A World City and Flexible Specialization: Restructuring of the Tokyo Metropolis. International Journal of Urban and Regional Research. vol 15, № 2, p. 269-284.

Gottdiener, Mark. 1997. The Theming of America. American Dreams, Media Fantasies and Themed Environments. Boulder: Westview Press, 206 p.

Graham, Stephen. 1992. The Role of Cities in Telecommunications Development.

Telecommunications Policy. vol. 16, $\mathrm{n}^{\circ}$ 3, p. 187-193.

Hein, Carola. 2008. Machi, Neighborhood and Small Town: the Foundation for Urban

Transformation in Japan. Journal of Urban History. vol 35-1, p. 75-107.

Hein, Carola \& Pelletier, Philippe (eds.). 2006. Cities, Autonomy and Decentralization in Japan, London: Routledge, $224 \mathrm{p}$.

Hirooka, Haruya. 2000. The Development of Tokyo's Rail Network. Japanese Railway and Transport Review. vol 23, p. 21-30.

Hoyaux, André-Frédéric. 1998. D’une conception archétypale de l'espace à une conception normative de l'habiter. Actes du colloque Dire, écrire et figurer l'espace. Tours : Presses Universitaires de Tours, $18 \mathrm{p}$.

Huat, Chua Beng. 2003. Life is not complete without shopping. Consumption culture in Singapore.

Singapour: Singapore University Press, 199 p. 
Hutcheon, Linda. 1986. The Politics of Post-Modernism: Parody and History. Cultural Critique, 1986, p. 179-207.

Jauss, Hans Robert. 1978. Pour une esthétique de la réception (traduit de l'allemand par C. Maillard). Paris : Gallimard, 336 p.

Jerde, Jon. 1988. A Philosophy for City Redevelopment. Meanjin. vol. 47, n 4, p. 609-613.

Jerde, Jon. 1998. Capturing the leisure Zeitgeist. Architectural Design. vol 68, nํ1-2, p. 69-71.

Joseph-Lester, Jaspar. 2010. Non-Relational Regimes of Urban Modernization. Journal of Visual Art Practice. vol 9, nº 2, p. 163-175.

Kärrholm, Mattias. 2012. Retailising Space: Architecture, Retail and the Territorialisation of Public Space . Farnham: Ashgate, $161 \mathrm{p}$.

Kawakami, Hidemitsu. 1988. Theory of the Urban Environmental Master Plan for Kawasaki City and Development Guiding Principle for the Supporting Planning Information System. Information Systems for Government and Business: Trends, Issues, Challenges. p. 95-103.

Koolhaas, Rem. 1995. Whatever Happened to Urbanism?. Design Quarterly, no 64, p. 28-31.

Kulke, Helmar. 2001. Entwicklungstendenzen suburbaner Einzelhandelslandschaften. In: Brake, Klaus (ed.). Suburbanisierung in Deutschland. Francfort: Springer, p. 57-69.

Kumata, Yoshinobu. 1990. Advanced Information Technologies of Urban Revitalization: the Campus City Kawasaki Plan. Cities. vol. 7, n 1, p. 75.

Leibowitz, Ed. 2004. The Great Mall of China. Time Express. no 102, p. 49-61.

Lukas, Scott. 2007. The Themed Space. Locating Culture, Nation and Self. Lanham: Rowman and Littlefield, $336 \mathrm{p}$.

Machimura, Takashi. 1992. The Urban Restructuring Process in Tokyo since the 1980s: Transforming Tokyo into a World City. International Journal of Urban and Regional Research. vol 16, $\mathrm{n}^{\mathrm{o}}$ 1, p. 114-128.

Miyamoto, Mitsuharu. 2012. A Triad Model for Promoting Start-Ups: Audition, Incubation and Venture-Capital Fundind: Evidence from Kawasaki City. Evolutionary and Institutional Economics Review. $\mathrm{n}^{\circ}$ 9, p. 75-99.

Morvan, Yoann. 2011. Kanal Istanbul : un "projet fou" au service d'ambitions politiques. Métropolitiques. [En ligne]. Disponible sur : http://www.metropolitiques.eu/Kanal-Istanbul-unprojet-fou-au.html (consulté le 23 septembre 2015).

Nédélec, Pascale. 2012. L'enclave fonctionnelle du Strip à Las Vegas : quand l'insularité façonne la ville. Espaces et Sociétés. ํㅜㄴ 150, p. 49-65.

Newstead, Anthony. 1989. Future information cities: Japan's vision. Futures, 21-3, p. 263-276.

Raz, Aviad. 2000. Domesticating Disney: Onstage Strategies in Tokyo Disneyland. The Journal of Popular Culture. vol 33, nº 4, p. 77-99.

Raz, Aviad. 2003. The Slanted Smile Factory: Emotion Management in Tokyo Disneyland. In Harper, Douglas \& Lawson, Helene (eds.). The Cultural Study of Work, Oxford: Rowman and Littlefield, p. 210-227.

Reinermann, Heinrich. 1986. International Design Competition for an Advanced Information City: Campus City Kawasaki. Speyer: Hochschule für Verwaltungswissenschaften, 32 p. 
Ritzer, George. 2001. The Modern Las-Vegas Casino Hotel: The Paradigmatic New Means of Consumption. Management. vol 3, nº 4, p. 83-99.

Roberts, Glanda. 2002. Pining Hopes on Angels: Reflections from an Aging Japan's Urban Landscape. In Goodman, Roger (ed.). Family and Social Policy in Japan: Anthropological Approaches. Cambridge: Cambridge University Press, p. 54-90.

Schencking, Charles. 2008. The Great Kanto Earthquake and the Culture of Catastrophe and Reconstruction in 1920s Japan. Journal of Japanese Studies. vol 34, no2, p. 295-331.

Soja, Edward. 1995. Postmodern urbanization: the six restructurings of Los Angeles. Postmodern Cities and Spaces, p. 125-137.

Sorensen, André. 1999. Land Readjustment, Urban Planning and Urban Sprawl in the Tokyo Metropolitan Area. Urban Studies. vol 36, p. 2333-2360.

Sorensen, André. 2000. Urban Renaissance as Intensification: Building Regulation and the Rescaling of Place Governance in Tokyo's High-Rise Manshon Boom. Urban Studies. vol 47, nº 3, p. 556-583.

Sorensen, André. 2002. The Making of Urban Japan: Cities and Planning from Edo to the $21^{\text {st }}$ century. London: Routledge, $400 \mathrm{p}$.

Sorensen, André. 2006. Liveable Cities in Japan: Population Ageing and Decline as Vectors of Change. International Planning Studies. vol 11, nº 3-4, p. 225-242.

Sterne, Jonathan. 1997. Sounds like the Mall of America: Programmed Music and the Architectonics of Commercial Space. Ethnomusicology. vol 41, no 1, p. 22-50.

Tiratsoo, Nick. 1990. Reconstruction, Affluence and Labour Politics: Coventry 1945-60, London: Routledge, $176 \mathrm{p}$.

Van Berkel, Rene. 2009. Quantitative Assessment of Urban and Industrial Symbiosis in Kawasaki, Japan. Environmental Science and Technology. vol 43, nº 5, p. 1271-1281.

Voyé, Liliane. 2003. Architecture et urbanisme post-modernes : une expression du relativisme contemporain ?. Revue européenne des sciences sociales. vol 41, nº 126, p. 117-124.

\section{NOTES}

1. Planning the future of Kawasaki as an information city, centre de documentation du service municipal d'urbanisme de Kawasaki. J'ai pu repérer ce document grâce aux indications données par Newstead, 1989.

2. Sur la stimulation auditive de la consommation par la création d'une connivence culturelle thématisée, voir : Sterne, 1997.

3. Sur ce sujet, outre les réflexions fondatrices d'Umberto Eco, voir : Dodds, 2003.

4. Sur cette dimension « glocale » des bars de la chaîne Starbucks, voir : Arsel \& Thompson, 2004.

5. Sur ce lieu, voir : Raz, 2000 ; Raz, 2003.

6. Sur les politiques d'industrialisation de la consommation à Singapour, voir : Huat, 2003.

7. Pour cet angle d'analyse des expressions architecturales et urbaines post-modernes, voir: Hutcheon, 1986.

8. Sur ce dernier quartier, voir : Morvan, 2011. 


\section{RÉSUMÉS}

Dans un centre-ville où une ancienne usine Toshiba constituait une friche industrielle coupant la ville en deux et où les flux de passagers des trains de banlieue arrivant de Tokyo se déversaient sans réellement donner à l'espace une consistance d'urbanité, les autorités municipales de Kawasaki décidèrent, à la fin des années 1990, de confier à deux architectes étrangers, Jon Jerde et Ricardo Bofill, le soin de travailler sur les ambiances urbaines. Les solutions retenues jouent toutes les deux sur la mise en scène d'un caractère méditerranéen et sur la construction de toutes pièces d'une atmosphère urbaine transplantée. L'objet du présent article est d'analyser comment ce décalage, créé dans la fiction culturelle d'un référent déplacé, reflète certaines tendances contemporaines de l'urbanisme post-moderne et thématisé, illustre le choix du pastiche comme voie de création d'urbanité face aux impasses de la foi en la puissance urbaine de la technologie, et invite à réfléchir sur les caractères mêmes de ce qui fonde l'urbanité.

In Downtown Kawasaki the presence of an industrial brownfield (ex-Toshiba factory) and the fact that commuters arriving from Tokyo just rushed towards their respective residential neighbourhoods combined in making the local characters of urbanity very fragile. Local authorities and two major real estate and commercial companies asked, at the end of the 1990s and following two decades of reflections on information technologies being a tool of urban revitalization, two foreign architects, Jon Jerde and Ricardo Bofill, to work on urban atmospheres. Both chose to build a pastiche urban scenography inspired by the Mediterranean. The object of this article is to analyse how such post-modern cultural fictions reflect present trends in themed urbanism, to reflect on pastiche as a tool of urbanity creation and to discuss what constitutes the very fundamental characters of urbanity.

\section{INDEX}

Keywords : Japan, Kawasaki, urbanity, architecture, town-planning, themed urbanism, urban revitalization

Mots-clés : Japon, Kawasaki, urbanité, architecture, projet urbain, urbanisme thématisé, revitalisation urbaine, post-industriel

\section{AUTEUR}

\section{DENIS BOCQUET}

Denis Bocquet est professeur d'histoire et théorie de l'architecture et de la ville à l'Ecole nationale supérieure d'architecture de Strasbourg (ENSAS/laboratoire AMUP). Ses recherches portent sur le lien entre formes construites, caractères de l'urbanité et représentations culturelles. denis.bocquet@strasbourg.archi.fr 